October 1998

PUPT-1816

hep-th/9810135

\title{
BTZ black holes and the near-horizon geometry of higher-dimensional black holes
}

\author{
Yuji SATOH \\ Joseph Henry Laboratories, Princeton University \\ Princeton, NJ 08544, USA
}

\begin{abstract}
We investigate the connection between the BTZ black holes and the near-horizon geometry of higher-dimensional black holes. Under mild conditions, we show that (i) if a black hole has a global structure of the type of the non-extremal ReissnerNordstrom black holes, its near-horizon geometry is $A d S_{2}$ times a sphere, and further (ii) if such a black hole is obtained from a boosted black string by dimensional reduction, the near-horizon geometry of the latter contains a BTZ black hole. Because of these facts, the calculation of the Bekenstein-Hawking entropy and the absorption cross-sections of scalar fields is essentially reduced to the corresponding calculation in the BTZ geometry under appropriate conditions. This holds even if the geometry is not supersymmetric in the extremal limit. Several examples are discussed. We also discuss some generalizations to geometries which do not have $A d S$ near the horizon.
\end{abstract}

\footnotetext{
${ }^{\dagger}$ ysatoh@viper.princeton.edu
} 


\section{Introduction}

The three dimensional black holes discovered by Bañados, Teitelboim and Zanelli (BTZ) have a very simple geometry which is locally three dimensional anti-de Sitter space $\left(A d S_{3}\right)$ [1]. In spite of this, it is known that they possess many characteristics of higher-dimensional black holes and serve as a useful toy model for black hole physics (for a review, see [2] ). Recent developments in string theory have revealed that they are more important than just as a simplified model: Some class of five- and four-dimensional black holes contains the BTZ black holes in the near-horizon region when they are lifted to the black strings in higher

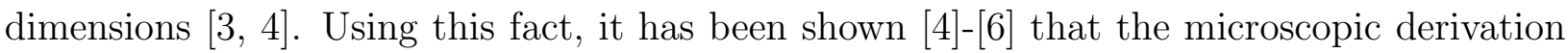
of the Bekenstein-Hawking entropy is reduced to the corresponding derivation for the BTZ black holes, which has been discussed in [7, [5, 81. I Such an argument has been extended to other black hole and black string geometries in [10-112]. Furthermore, by carefully looking at the calculation of the greybody factors for the $D=5$ and 4 black holes [13] and by taking into account their connection to the BTZ black holes, one realizes that the greybody factors effectively come from the near-horizon BTZ geometry. (For details, see the discussion in section 4). Some greybody factors in these cases have also been derived [14, 15] using the near-horizon BTZ $\left(A d S_{3}\right)$ geometry and the AdS/CFT correspondence [16]. The AdS/CFT correspondence is particularly interesting because it might provide the fundamental quantum theory of the black holes via the near-horizon BTZ black holes.

In addition to these advances, for a large class of non-extremal black hole and $p$-brane geometries it has been shown in 17 that the scalar wave equations possess features closely related to $A d S_{3}(S L(2, R))$ and that the greybody factors take the form expected of a CFT in some parameter region. These suggest that the connections among black holes, the $\mathrm{BTZ}\left(A d S_{3}\right)$ geometry and some CFT might be extended to more general black objects than the above $D=5$ and 4 black holes.

In this paper, we investigate the connection between the BTZ black holes and the nearhorizon geometry of higher-dimensional black holes and $p$-branes. In section 2 , under mild conditions we show that (i) if a black hole has a global structure of the type of the nonextremal Reissner-Nordstrom black holes, its near-horizon geometry becomes $A d S_{2}$ times a sphere, and (ii) if such a black hole is obtained from a boosted black string by dimensional reduction, the near-horizon geometry of the latter contains a BTZ black hole. In section 3, we show that the entropy counting of these black holes is reduced to that of the corresponding BTZ black holes under appropriate conditions. In section 4, we discuss the greybody factors for scalar fields. We show that (i) the scalar wave equations are identical to the $s$-wave equation on the BTZ geometry (this holds in all the cases discussed in [17), and further (ii)

\footnotetext{
${ }^{1}$ For the unsolved problems in $[7,5,8]$, see [9].
} 
the greybody factors essentially come from the near-horizon BTZ geometry and take the form expected of a CFT in some parameter region. In section 5, we give several examples. They include the well-known $D=5$ and 4 black holes with three and four charges respectively, the four-dimensional $N=2$ black holes and dyonic dilaton black holes in generic dimension. These last dyonic black holes include the Reissner-Nordstrom black holes themselves as special cases. In section 6, we generalize the results in the preceding sections to geometries which do not contain $A d S$ near the horizon. In section 7, we conclude with a summary and a discussion of the future directions. The appendix contains some technical details about the near-horizon geometry of $p$-branes (A.1), $A d S_{2}, A d S_{3}$ and the BTZ geometry (A.2) and scalar wave equations and dimensional reduction (A.3).

\section{Geometries with two horizons}

In this section, we consider geometries with a global structure of the type of the non-extremal Reissner-Nordstrom black holes.

\section{1 near-horizon geometries of $p$-brane solutions}

As a preliminary, we study a geometry with the metric of the form

$$
\begin{aligned}
d s_{p}^{2} & =\left(\frac{r}{q}\right)^{\alpha d}\left[-f(r) d t^{2}+d y^{i} d y^{i}\right]+\left(\frac{q}{r}\right)^{2 \beta} f^{-1}(r) d r^{2}, \\
f(r) & =1-\frac{r_{0}^{d}}{r^{d}}
\end{aligned}
$$

where $i=1, \ldots, p$. $p$-brane solutions typically contain this type of geometry near the horizon. $r_{0} \rightarrow 0$ corresponds to their extremal limit.2 We are interested in the case in which this becomes (locally) $A d S$. Since a Lorentzian space becomes locally $A d S$ if and only if it has negative constant curvature, (2.1) is locally $A d S$ only when the tensor

$$
K_{a b c d}=R_{a b c d}-k\left(g_{a c} g_{b d}-g_{a d} g_{b c}\right)
$$

vanishes for a negative constant $k$. It is straightforward to calculate this for (2.1). We display the result in the appendix. We then find that for $r_{0}=0$ the geometry is $A d S_{p+2}$ if $\beta=1$ $\left(\alpha, d\right.$ are arbitrary). Setting $(r / q)^{\alpha d}=(\omega / c)^{2}$ with $c$ a constant, the metric is expressed by Poincarè coordinates like (A.8). However, for $r_{0} \neq 0$ it cannot be $A d S$ in generic dimension. The exceptional cases are $p=0$ and 1: For $p=0$ we have $A d S_{2}$ when $(\alpha, \beta)=(1,1),(2,1)$. By a coordinate transformation $\left(r / r_{0}\right)^{d}=z$, the metric takes the form of the type (A.5) for $\alpha=1$ and (A.6) for $\alpha=2$. For $p=1$ we have $A d S_{3}$ only when $(\alpha, \beta)=(1,1)$. By a transformation $\left(r / r_{0}\right)^{d}=(\rho / l)^{2}$, the metric is brought to the form of the type (A.9).

\footnotetext{
${ }^{2}$ The near-horizon geometries of some class of extremal $p$-branes have been discussed in detail in 18 , 19.
} 
The $A d S_{3}$ geometry of the type (A.8) typically appears for extremal black holes and $p$-branes whereas that of the type (A.9) in non-extremal cases. The solutions to the scalar wave equation in each coordinate system have recently been studied in detail [20, 21] in the context of the AdS/CFT correspondence [16]. It has also been observed that the transition from (A.8) to (A.9) causes the thermalization of the associate quantum states [22, 21, 15]. Mathematically, these two coordinate systems are naturally related to the representations of $S L(2, R)$ in the parabolic and hyperbolic basis, respectively. The scalar wave equation is nothing but the Laplace equation on $S L(2, R)$ and its solutions correspond to the matrix elements of the representations. They have been well-classified in terms of the representation theory. The transition from (A.8) to (A.9) corresponds to a change of the basis and the transition functions (Bogoliubov coefficients) are also well-studied. For a review, see, e.g., 23, 24,

\section{2 near-horizon geometry of a non-extremal Reissner-Nordstrom type black hole}

In the following, we consider a spherically symmetric geometry in $D(=d+3)$ dimensions,

$$
d s^{2}=-F_{1}(r) f(r) d t^{2}+F_{2}(r)\left[f^{-1}(r) d r^{2}+r^{2} d \Omega_{d+1}^{2}\right]
$$

where $d \Omega_{d+1}^{2}$ is the metric of the unit $(d+1)$-sphere. If $F_{i}(i=1,2)$ are arbitrary, this is the most general form of a spherically symmetric geometry. Here we focus on the case in which (2.3) expresses a non-extremal Reissner-Nordstrom type black hole, namely, in which it has two horizons whose $(t, r)$-part tends to become Rindler space near the horizon (up to an overall sign),

$$
d s_{\text {Rindler }}^{2}=-\kappa^{2} \rho^{2} d t^{2}+d \rho^{2},
$$

with $\kappa$ a constant. We further concentrate on the case where the outer and inner horizon are located at $r=r_{0}$ and $r=0$ respectively. Then $F_{i}\left(r_{0}\right)$ should be non-vanishing so that the

geometry contains Rindler space as $r \rightarrow r_{0}$. By a coordinate transformation $\rho \sim \sqrt{r_{0}\left(r-r_{0}\right)}$, we confirm that $(t, r)$-part becomes of the form (2.4). Similarly, the requirement for $r \rightarrow 0$ gives several conditions. Firstly, since the radius of $S^{d+1}$ should be regular, we have

$$
F_{2}(r) \rightarrow\left(\frac{r_{2}}{r}\right)^{2} \quad \text { as } r \rightarrow 0
$$

with $r_{2}$ some constant. In addition, $F_{1}$ should satisfy

$$
F_{1}(r) \rightarrow\left(\frac{r}{r_{1}}\right)^{2 d} \quad \text { as } r \rightarrow 0
$$

Under these conditions, the geometry actually takes the form (2.4) times $S^{d+1}$ as $r \rightarrow 0$ by the coordinate transformation $\rho \sim r_{0}\left(r / r_{0}\right)^{d / 2}$. For later convenience, we rescale $t$ so that 
$r_{1}=r_{2}=q$ and rewrite $F_{i}(r)$ by $F_{1}=H_{1}^{-2}$ and $F_{2}=H_{2}^{2 / d}$. Consequently, the metric is expressed as

$$
d s^{2}=-H_{1}^{-2}(r) f(r) d t^{2}+H_{2}^{2 / d}(r)\left[f^{-1}(r) d r^{2}+r^{2} d \Omega_{d+1}^{2}\right]
$$

where

$$
H_{i}\left(r_{0}\right) \neq 0, \quad H_{1,2}(r) \rightarrow\left(\frac{q}{r}\right)^{d} \quad \text { as } r \rightarrow 0 .
$$

In the 'near-horizon' region where $H_{i}(r)$ are well-approximated by $(q / r)^{d}$, the geometry becomes

$$
\tilde{d s}^{2} \sim\left(\frac{r}{q}\right)^{2 d}\left[1-\left(r_{0} / r\right)^{d}\right] d t^{2}+\left(\frac{q}{r}\right)^{2} \frac{d r^{2}}{1-\left(r_{0} / r\right)^{d}}+q^{2} d \Omega_{d+1}^{2}
$$

This corresponds to the $(\alpha, \beta)=(2,1)$ case for $p=0$ in the previous section and thus the near-horizon geometry is $A d S_{2} \times S^{d+1}$. Here we remark that the near-horizon region is not necessarily restricted to $r \sim 0$, which is obvious from (2.8). For example, we can take $H_{i}$ to be a product of harmonic functions,

$$
H_{1}(r)=H_{2}(r)=\prod_{i}\left(1+\frac{r_{i}^{d}}{r^{d}}\right)^{a_{i}}, \quad r_{i}^{d}=r_{0}^{d} \sinh ^{2} \delta_{i}
$$

with $\sum a_{i}=1$. In this case, the 'near-horizon' region is given by $r_{i} \gg r$.

\section{3 near-horizon geometry of the lifted black string}

If a $D$-dimensional black hole solution is obtained by dimensional reduction from a $(D+1)$ dimensional solution, the corresponding geometry represents a black string. Here we are interested in a boosted black string geometry,

$$
\begin{aligned}
d s_{D+1}^{2}=\hat{H}_{1}^{-1}(r)\left[-d t^{2}\right. & \left.+d x^{2}+\left(r_{0} / r\right)^{d}(\cosh \sigma d t-\sinh \sigma d x)^{2}\right] \\
& +\hat{H}_{2}^{2 / d}(r)\left[f^{-1}(r) d r^{2}+r^{2} d \Omega_{d+1}^{2}\right]
\end{aligned}
$$

with $\sigma$ the boost angle in $(t, x)$-part. By the dimensional reduction with respect to $x$, (2.11) reduces to (2.7) if

$$
\hat{H}_{1}=H_{1}\left[H_{1} h_{\sigma}^{-1}\right]^{d /(d+2)}, \quad \hat{H}_{2}=H_{2}\left[H_{1} h_{\sigma}^{-1}\right]^{d /(d+2)},
$$

where $h_{\sigma}(r)=1+\left(r_{\sigma} / r\right)^{d}$ and $r_{\sigma}^{d}=r_{0}^{d} \sinh ^{2} \sigma$.

${ }^{3}$ This holds even in the extremal limit $r_{0} \rightarrow 0$. The arguments in the following are also valid in this limit if it is not singular. 
In the 'near-horizon' region where $H_{i}(r) \sim(q / r)^{d}$ and $h_{\sigma}(r) \sim\left(r_{\sigma} / r\right)^{d}$, t the geometry becomes

$$
\begin{gathered}
\widetilde{d s}_{D+1}^{2} \sim\left(\frac{r}{\hat{q}}\right)^{d}\left[-d t^{2}+d x^{2}+\left(r_{0} / r\right)^{d}(\cosh \sigma d t-\sinh \sigma d x)^{2}\right] \\
+\left(\frac{\hat{q}}{r}\right)^{2} \frac{d r^{2}}{1-\left(r_{0} / r\right)^{d}}+\hat{q}^{2} d \Omega_{d+1}^{2}
\end{gathered}
$$

where

$$
\hat{q}=q\left(q / r_{\sigma}\right)^{d /(d+2)}
$$

We find that this corresponds to the $(\alpha, \beta)=(1,1)$ case for $p=1$ in section 2.1 and hence the near-horizon geometry is locally $A d S_{3} \times S^{d+1}$. Moreover, when $x$ is periodic, i.e., $x \sim x+2 \pi R$, this is nothing but the BTZ geometry [1]. Indeed, by the coordinate transformation [3, 4, 6]

$$
\begin{aligned}
\frac{\rho^{2}}{R^{2}} & =\left(\frac{r}{\hat{q}}\right)^{d}+\left(\frac{r_{0}}{\hat{q}}\right)^{d} \sinh ^{2} \sigma, \\
\varphi & =\frac{x}{R}, \quad \tau=\frac{l}{R} t, \quad l=\frac{2}{d} \hat{q},
\end{aligned}
$$

the geometry $(2.13)$ takes the standard form of the BTZ metric

$$
\begin{aligned}
d s_{B T Z}^{2} & =-N(\rho) d \tau^{2}+\rho^{2}\left(d \varphi-\frac{\rho_{+} \rho_{-}}{l \rho^{2}} d \tau\right)^{2}+N^{-1}(\rho) d \rho^{2} \\
N(\rho) & =\frac{\left(\rho^{2}-\rho_{+}^{2}\right)\left(\rho^{2}-\rho_{-}^{2}\right)}{l^{2} \rho^{2}}
\end{aligned}
$$

where

$$
\rho_{+}^{2}=R^{2}\left(r_{0} / \hat{q}\right)^{d} \cosh ^{2} \sigma, \quad \rho_{-}^{2}=R^{2}\left(r_{0} / \hat{q}\right)^{d} \sinh ^{2} \sigma
$$

Therefore if the black hole (2.7) is obtained from a boosted black string, the near-horizon geometry of the latter contains a BTZ black hole.

\section{$3 \quad$ Black hole entropy}

In this section, we will show that under some conditions the Bekenstein-Hawking entropy of the Reissner-Nordstrom type black holes (2.7) is obtained from the entropy of the BTZ black holes contained in the lifted black string geometries.

\footnotetext{
${ }^{4}$ When $\hat{H}_{i}$ becomes independent of $r_{\sigma}$, the condition $h_{\sigma} \sim r_{\sigma}^{d} / r^{d}$ is not necessary. See the examples discussed later.
} 
To see this, let us first note that the entropy of the BTZ black hole (2.16) is given by

$$
S_{B T Z}=\frac{A_{3}}{4 G_{3}}=\frac{\pi R}{2 G_{3}}\left(\frac{r_{0}}{\hat{q}}\right)^{d / 2} \cosh \sigma
$$

where $A_{D^{\prime}}$ and $G_{D^{\prime}}$ are the area of the outer horizon and the Newton constant in $D^{\prime}$ dimensions respectively. We have used $A_{3}=2 \pi \rho_{+}$. On the other hand, the entropy associated to the geometry (2.7) is

$$
S_{D}=\frac{A_{D}}{4 G_{D}}=\frac{\omega_{d+1}}{4 G_{D}}\left[r_{0} H_{2}^{1 / d}\left(r_{0}\right)\right]^{d+1},
$$

with $\omega_{d+1}$ the volume of the unit $(d+1)$-sphere.

To compare these two expressions, we need to know the relation between the Newton constants in different dimensions. In general, under dimensional reduction from $D^{\prime}+p$ dimensions to $D^{\prime}$ dimensions, the Newton constants in the effective action change as

$$
\frac{1}{G_{D^{\prime}+p}}=\frac{V_{p}}{G_{D^{\prime}}}
$$

where $V_{p}$ is the volume of the compactified space. This is valid when the metric is independent of the compactified coordinates. Thus we get $G_{D+1}=G_{D} / 2 \pi R$ when we lift the black hole to the black string. Furthermore, since the black string geometry factorizes into the BTZ geometry times $S^{d+1}$ in the near-horizon region, the Ricci scalar in the Lagrangian also splits into two parts. Hence we obtain a similar relation to (3.3); $G_{D+1}=G_{3} /\left(\hat{q}^{d+1} \omega_{d+1}\right)$. Combining these, one finds that

$$
\frac{1}{G_{D}}=\frac{2 \pi R}{\hat{q}^{d+1} \omega_{d+1} G_{3}} .
$$

Substituting this into (3.2) yields

$$
\begin{aligned}
S_{D} & =\gamma S_{B T Z} \\
\gamma & =\left(\frac{r_{0}}{\hat{q}}\right)^{1+d / 2} H_{2}^{(d+1) / d}\left(r_{0}\right) \cosh ^{-1} \sigma .
\end{aligned}
$$

Thus the two entropies agree when

$$
\gamma \sim 1
$$

This conditions is typically satisfied when the radii of the outer and inner horizon are close and $r_{\sigma} \gg r_{0}(\sigma \gg 1)$. In fact, this means that $r_{0} H_{2}^{1 / d}\left(r_{0}\right) \sim \lim _{r \rightarrow 0} r H_{2}^{1 / d}(r) \sim q$. Using (2.14), we find that

$$
\hat{q} \sim r_{0}\left[H_{2}^{2(d+1) / d}\left(r_{0}\right) \sinh ^{-2} \sigma\right]^{1 /(d+2)}
$$


and (3.6) is satisfied. In addition, it may be useful to evaluate $\gamma$ for the example given in (2.10). It is given by

$$
\gamma=\prod_{i}\left(\frac{\cosh \delta_{i}}{\sinh \delta_{i}}\right)^{2 a_{i} \frac{d+1}{d}} \frac{\sinh \sigma}{\cosh \sigma} .
$$

Thus $S_{D}$ is obtained from $S_{B T Z}$, e.g., when $\delta_{i}, \sigma \gg 1$, namely, the charges $r_{i}, r_{\sigma}$ are large enough or the geometry is near the extremal limit $r_{0} \rightarrow 0 ; \delta_{i}, \sigma \rightarrow \infty$. We remark that when

$$
2 a_{j}(d+1)=d, \quad \sigma=\delta_{j},
$$

for some $j, \hat{H}_{i}$ and $\hat{q}$ become independent of $\sigma=\delta_{j}$ and we do not need the condition for $r_{\sigma}$.

For the BTZ black holes, the microscopic derivation of the Bekenstein-Hawking entropy has been discussed in [7, 5, 8]. Our argument here indicates that the microscopic derivation of the entropy of the Reissner-Nordstrom type black holes in (2.7) is reduced to the corresponding derivation in [7, 5, 8] under appropriate conditions.

\section{Greybody factors}

We have discussed the Bekenstein-Hawking entropy. In this section, we discuss another important feature of black holes, i.e., greybody factors. We will find that they essentially come from the near-horizon BTZ geometry under mild conditions.

We begin with a brief review of the results of [17]. In [17], the propagation of minimally coupled massless scalars in various non-extremal black hole and $p$-brane geometries has been discussed. There it has been shown that by expanding a scalar field as $\Phi=\sum e^{-i \omega t} \phi_{m}(r) \chi_{m}$, the near-horizon equation for the $m$-th partial wave takes the form

$$
\left[\left\{z(z-1) \partial_{z}\right\}^{2}-z(z-1)\left\{\frac{\nu_{+}^{2}}{z-1}-\frac{\nu_{-}^{2}}{z}+\frac{\Lambda}{d^{2}}\right\}\right] \phi_{m}=0 .
$$

Here, $\chi_{m}$ is the eigenfunction of the Laplacian on $S^{d+1}$ with the eigenvalue $-\Lambda=-m(m+d)$; $\nu_{ \pm}=-i \beta_{ \pm} \omega / 4 \pi ; \omega$ is the frequency; $\beta_{ \pm}$are the periods of the imaginary time associated to the outer and inner horizon (when the geometry does not have an inner horizon, $\beta_{-}=0$ ). In addition, the above equation has been identified with the scalar wave equation in $S L(2, R)$ $\left(A d S_{3}\right)$. One finds this as follows. The eigenvalue equation of the Laplace operator on $S L(2, R)$ is given by

$$
\left[\frac{1}{\sqrt{-g}} \partial_{\mu} \sqrt{-g} g^{\mu \nu} \partial_{\nu}-4 j(j+1) l^{-2}\right] \Phi=0,
$$

where $j$ is the $S L(2, R)$ spin and $l$ is a parameter in terms of which the scalar curvature is written as $-6 l^{-2}$. Here we use the coordinate system in (A.9) and make a change of variables 
$z=(r / l)^{2}$. As $z \rightarrow 1$ and 0 , the geometry approaches Rindler space. We further rescale $\theta_{ \pm}$as $\tilde{\theta}_{ \pm}=\left(\tilde{\beta}_{ \pm} / 2 \pi l\right) \theta_{ \pm}$so that their imaginary periods associated to the Rindler space-like regions become $\beta_{ \pm}$. By a separation of variables $\Phi=\sum \exp \left(i \omega_{+} \tilde{\theta}_{+}+i \omega_{-} \tilde{\theta}_{-}\right) \phi(r)$, the radial equation for $\phi$ takes the same form as (4.1) under the identifications

$$
\beta_{ \pm} \leftrightarrow \tilde{\beta}_{ \pm}, \quad-(1+m / d) \leftrightarrow j, \quad|\omega| \leftrightarrow\left|\omega_{ \pm}\right|
$$

This means that the near-horizon wave equations for the various black objects are regarded as those in the $S L(2, R)$ background.

Now we show that, more precisely, (4.1) is naturally identified with the $s$-wave equation on the BTZ background. The point is the last identification in (4.3). For definiteness, we fix the signs and take it to be $\omega=\omega_{ \pm}$. This indicates that we are dealing with the wave function $\Phi=\sum \phi(r) \exp \left[i \omega\left(\tilde{\theta}_{+}+\tilde{\theta}_{-}\right)\right]$. Here let us recall that the time and angle coordinate of the BTZ metric (2.16) are given by (A.12). In terms of the periods of the imaginary time associated to the two horizons,

$$
\tilde{\beta}_{ \pm}=\frac{2 \pi l^{2} \rho_{ \pm}}{\rho_{+}^{2}-\rho_{-}^{2}}
$$

they are given by

$$
\left(\begin{array}{c}
\tau \\
l \varphi
\end{array}\right)=\frac{1}{2 \pi l}\left(\begin{array}{cc}
\tilde{\beta}_{+} & \tilde{\beta}_{-} \\
\tilde{\beta}_{-} & \tilde{\beta}_{+}
\end{array}\right)\left(\begin{array}{c}
\theta_{+} \\
\theta_{-}
\end{array}\right)
$$

Therefore, $\tilde{\theta}_{+}+\tilde{\theta}_{-}=\tau$ and $\Phi=\sum \phi(r) \exp \left[i \omega\left(\tilde{\theta}_{+}+\tilde{\theta}_{-}\right)\right]=\left.\sum \phi(r) \exp (i \omega \tau+i n \varphi)\right|_{n=0}$. Namely, the wave equation is the $s$-wave equation on the BTZ background.

Let us turn to the wave equation of a massless scalar in the geometry (2.7). Using the same separation of variables as for (4.1), one obtains the radial equation,

$$
\left[\left\{\left(H_{2} / H_{1}\right) f r^{d+1} \partial_{r}\right\}^{2}+H_{2}^{2(d+1) / d} r^{2(d+1)} \omega^{2}-\left(H_{2} / H_{1}\right)^{2} f r^{2 d} \Lambda\right] \phi_{m}(r)=0 .
$$

In the near-horizon region where $H_{1} \sim H_{2} \sim(q / r)^{d}$, a change of variables $\left(r / r_{0}\right)^{d}=z$ yields the wave equation of the form (4.1) with $\nu_{+}^{2} \sim \nu_{-}^{2}=-\left(q / r_{0}\right)^{2(d+1)}\left(r_{0} \omega / d\right)^{2}$. Thus it is identical to the $s$-wave equation on the BTZ background as discussed above.

In section 2, we saw that the near-horizon geometry of (2.7) is not $A d S_{3} \times S^{d+1}$ but $A d S_{2} \times S^{d+1}$. Then why does the wave equation in the geometry (2.7) become the $s$-wave equation in the BTZ background? One can understand this as follows. When the black hole is lifted to the black string, the near horizon geometry of the latter contains the BTZ

${ }^{5}$ To directly compare the wave equation from the near-horizon BTZ geometry with (4.6) near the horizon, in addition to (4.3) we need to take into account the rescale of the time coordinate in (2.15), i.e., the rescale of the frequency $\omega \rightarrow(l / R) \omega$. 
geometry. In addition, the radial wave equation is invariant under the dimensional reduction if the wave function is independent of the reduced coordinate (see, the appendix). Thus the near-horizon equation for (2.7) is the same as the corresponding equation for the black string and hence it becomes the wave equation in the BTZ geometry. Moreover, since the reduced coordinate is nothing but the angle coordinate of (2.16), being independent of the reduced coordinate means taking the $s$-waves in terms of the BTZ geometry.

Having established the relation between (4.6) and (4.1), similarly to [25, 17] one can show that the greybody factors take the form expected of a CFT at low energy. To see this, we need only mild assumptions such as asymptotic flatness $\left(H_{i} \rightarrow 1\right.$ as $\left.r \rightarrow \infty\right), q / r_{0} \gg 1$ ('large charge') and so on. In particular, when the geometry is given by (2.10), the argument is almost the same as in 17.0

Furthermore, we realize that the greybody factors essentially come form the near-horizon BTZ geometry. This is because (i) the essence of the calculation of the absorption crosssections is the matching between the wave functions in the near-horizon and the asymptotically flat region, (ii) the non-trivial energy-dependence (greybody factors) comes from the former and (iii) near the horizon the wave equation (4.6) becomes the equation in the BTZ geometry contained in the lifted black string geometry. Thus the calculation of the greybody factors for the geometry (2.7) becomes essentially the same as the corresponding calculation for the BTZ geometry discussed in [26]. It may be worth noting that the greybody factors obtained in [26] take the form expected of a CFT only for the $s$-waves.

Therefore, we see that the BTZ black holes play an important role in the study of Hawking radiation for the Reissner-Nordstrom type geometry (2.7). Taking into account the result in appendix A.3, we also see that the argument in this section can be generalized to the charged scalar case.

It is subtle to explain the BTZ structure in (4.6) directly in terms of the near-horizon geometries. When we dimensionally reduce (2.16) (in string frame) with respect to $\varphi$, we obtain the metric

$$
d s_{2}^{2}=-N(\rho) d t^{2}+N^{-1}(\rho) d \rho^{2}
$$

with the dilaton $e^{-2 \Psi}=\rho$. We cannot absorb the dilaton and go to Einstein frame since we are in two dimensions. By a coordinate transformation $z=\left(\rho^{2}-\rho_{-}^{2}\right) / \Delta^{2}$ with $\Delta^{2}=\rho_{+}^{2}-\rho_{-}^{2}$, the metric becomes

$$
d s_{2}^{2}=-\left(\frac{\Delta}{l}\right)^{2} \frac{z(z-1)}{z+\left(\rho_{-} / \Delta\right)^{2}} d t^{2}+\frac{l^{2}}{4} \frac{d z^{2}}{z(z-1)} .
$$

${ }^{6}$ According to the form of $H_{i}$, more precise approximation is of course possible as extensively studied in the literature, e.g., [13]. 
This is not $A d S_{2}$ contrary to the near-horizon geometry of (2.7). It approaches $A d S_{2}$ when $z \ll \rho_{-}^{2} / \Delta^{2}$. This corresponds to $\rho \rightarrow \rho_{-}$or $\rho_{-} \rightarrow \rho_{+}$with $z$ fixed. In the latter case, it follows from (4.5) that $\varphi \propto \theta_{L}$ where $\theta_{L}=\left(\theta_{+}+\theta_{-}\right) / l$ is an analog of an Euler angle associated to the left $S L(2, R)$. Then the above dimensional reduction becomes that discussed in [27, 28] which relates $A d S_{3}$ to $A d S_{2}$ (see also the appendix). The argument here is essentially the same as in [28] in the extremal case. Though the scalar wave equation in (4.7) does not take the form (4.1), the tachyon wave equation $\left[\left(\sqrt{-g} e^{-2 \Psi}\right)^{-1} \partial_{\mu} \sqrt{-g} e^{-2 \Psi} g^{\mu \nu} \partial_{\nu}-\mu^{2}\right] \Phi=0$ does.

\section{$5 \quad$ Examples}

We argued that the entropy and the greybody factors of the Reissner-Nordstrom type black holes (2.7) effectively come from the near-horizon BTZ black holes. In this section, we give several examples.

The first examples of the geometry of the type (2.7) are the $D=5$ and 4 black holes in string theory. The metrics are given respectively by (see, e.g., 29])

$$
H_{1}^{(5)}=H_{2}^{(5)}=\prod_{i=1}^{3}\left(1+\frac{r_{i}^{2}}{r^{2}}\right)^{1 / 3}, \quad H_{1}^{(4)}=H_{2}^{(4)}=\prod_{i=1}^{4}\left(1+\frac{r_{i}}{r}\right)^{1 / 4}
$$

where $r_{i}^{d}=r_{0}^{d} \sinh ^{2} \delta_{i}$. To get the corresponding black string geometry, we set, e.g., $r_{1}=r_{\sigma}$ in $(2.12$ ) so that

$$
\hat{H}_{1,2}^{(5)}=\prod_{i=2}^{3}\left(1+\frac{r_{i}^{2}}{r^{2}}\right)^{1 / 2}, \quad \hat{H}_{1,2}^{(4)}=\prod_{i=2}^{4}\left(1+\frac{r_{i}}{r}\right)^{1 / 3} .
$$

Note that $r_{\sigma}$-dependence has disappeared.

In this case, the microscopic derivation of the Bekenstein-Hawking entropy has been discussed in [4]-6] using the near-horizon BTZ geometry. Since the condition (3.9) holds, the condition $\gamma \sim 1$ in section 3 is satisfied irrelevantly to $r_{\sigma}$ if $\delta_{j} \gg 1(j=2,3$ for $D=5$ and $j=2,3,4$ for $D=4$ ). Note that this includes the dilute gas region $r_{0}, r_{1} \ll r_{j}$.

As for the greybody factors, the fact that they essentially come from the near-horizon BTZ geometry has already been implied in the literature: we have only to repeat the discussion in the previous section. This was explicitly confirmed in the $D=5$ case by replacing the near-horizon geometry with the BTZ geometry [30. We also find that the $S L(2, R)$ structure of the wave equations observed in [31] is due to the near-horizon BTZ geometry. The greybody factors in these cases have been derived using the AdS/CFT correspondence [14, 15. 
The next example is a class of the black holes in the four-dimensional $N=2$ supergravity [32]. The geometry is given by

$$
H_{1}^{N=2}=H_{2}^{N=2}=\left(h_{g} d^{A B C} h_{A} h_{B} h_{C}\right)^{1 / 4},
$$

where $A, B, C=1, \ldots, n_{v} ; h_{g, A}(r)=b_{g, A}\left(1+r_{g, A} / r\right) ; r_{g, A}=r_{0} \sinh ^{2} \delta_{0, A} ; b_{g, A}$ are constants satisfying $b_{g} d^{A B C} b_{A} b_{B} b_{C}=1 ; n_{v}$ is the number of the vector multiplets (not including the universal one); and $d^{A B C}$ are the topological intersection numbers of the Calabi-Yau manifold. The corresponding black string solution is obtained by setting $r_{\sigma}=r_{g}$ [12];

$$
\hat{H}_{1,2}^{N=2}=\left(b_{g} d^{A B C} h_{A} h_{B} h_{C}\right)^{1 / 3} .
$$

In this case, the microscopic entropy counting using the near-horizon BTZ geometry has been discussed in [12]. Similarly to [13, 25, 17], one can discuss the greybody factors. The entropy counting for five-dimensional $N=2$ extremal black holes has also been discussed in [12.

Now we discuss new examples. Here we consider a class of dyonic dilaton black holes [33]. The metric is given by (2.7) withl]

$$
H_{1}^{\mathrm{Dy}}=H_{2}^{\mathrm{Dy}}=\left[\left(1+\frac{r_{1}^{d}}{r^{d}}\right)^{\alpha_{1}}\left(1+\frac{r_{2}^{d}}{r^{d}}\right)^{\alpha_{2}}\right]^{1 / 2},
$$

where $\alpha_{i}$ are some parameters satisfying $\alpha_{1}+\alpha_{2}=2$. This includes the Reissner-Nordstrom black holes in generic dimension as the special case $r_{1}=r_{2}$. Note that generically they are not supersymmetric even in the extremal limit $r_{0} \rightarrow 0$. We further focus on the case of $\alpha_{1}=d /(d+1), \alpha_{2}=(d+2) /(d+1)$. $d=1$ and 2 give special cases of (5.1). By setting $r_{\sigma}=r_{1}$ in (2.12), we obtain the lifted black string geometry,

$$
\begin{aligned}
d s_{b s}^{2}=\left(1+\frac{r_{2}^{d}}{r^{d}}\right)^{-1}[ & \left.-d t^{2}+d x^{2}+\left(r_{0} / r\right)^{d}(\cosh \sigma d t-\sinh \sigma d x)^{2}\right] \\
& +\left(1+\frac{r_{2}^{d}}{r^{d}}\right)^{2 / d}\left[f^{-1}(r) d r^{2}+r^{2} d \Omega_{d+1}^{2}\right],
\end{aligned}
$$

which corresponds to $\hat{H}_{1,2}^{\mathrm{Dy}}=1+\left(r_{2} / r\right)^{d}$. This is nothing but the boosted geometry of the non-dilatonic black string solution by [19]. The entropy counting for this class of dyonic black holes is again reduced to that of the BTZ black hole. Since the condition (3.9) again holds, $\gamma \sim 1$ is satisfied if $r_{2} \gg 1$. The greybody factors for these geometries have been discussed in [17].

\footnotetext{
${ }^{7}$ We use slightly different notations from those in [17]; the relation is $r^{d} \leftrightarrow r^{d}+\eta_{0}, r_{0}^{d} \leftrightarrow 2 \eta_{0}$ and so on.

8 Most of the discussion in the previous sections formally holds for generic $\alpha_{i}$. However, the physical interpretation is unclear unless we explicitly construct the lifted black string solution.
} 


\section{$6 \quad$ Generalizations to other black holes and $p$-branes}

The argument in the previous sections can be generalized to some cases in which the nearhorizon geometry is not directly related to $A d S$.

Let us first note that because of the relation between the Newton constants in different dimensions (3.3), the Bekenstein-Hawking entropy is invariant under dimensional reduction;

$$
\frac{A_{D}}{4 G_{D}}=\frac{A_{D+p}}{4 G_{D+p}} .
$$

In addition, the radial wave equation is also invariant (as discussed in the appendix). On the other hand, the near-horizon geometry is not; even if a geometry contains $A d S$ near the horizon, generically the near-horizon geometry connected by dimensional reduction does not contain $A d S$. These allow us to reduce the discussion on the entropy and greybody factors to that for the BTZ black holes even for the geometries without near-horizon $A d S$.

In the following, we will focus on a class of $p$-branes in $D$ dimensions [34], f]

$$
\begin{gathered}
d s_{p B}^{2}=H^{\alpha}(r)\left(H^{-N}(r)\left[-f(r) d t^{2}+d y^{i} d y^{i}\right]+f^{-1}(r) d r^{2}+r^{2} d \Omega_{d+1}^{2}\right), \\
H(r)=1+\frac{Q}{r^{d}}, \quad \alpha=\frac{p+1}{d+p+1} N,
\end{gathered}
$$

where $i=1, \cdots, p, D=d+p+3$ and $N$ is some parameter. The extremal case $\left(r_{0}=0\right)$ is supersymmetric if and only if $N$ is an integer. For later use, we introduce another parameter

$$
\xi=1+\frac{1}{d}-\frac{N}{2} .
$$

The dimensional reduction with respect to $y^{i}$ connect the geometries with different $p$ but $N$ and $\xi$ are invariant. The Reissner-Nordstrom black holes discussed in the previous section are included in (6.2) and correspond to $\xi=0, p=0$.

We first discuss a class of magnetically charged black holes by [35], which is a special case of (6.2) with $\xi=1 / 2, p=0$;

$$
d s_{\mathrm{mag}}^{2}=-\left(1+\frac{Q}{r^{d}}\right)^{-(d+2) /(d+1)} f(r) d t^{2}+\left(1+\frac{Q}{r^{d}}\right)^{(d+2) / d(d+1)}\left[f^{-1}(r)+r^{2} d \Omega_{d+1}^{2}\right] .
$$

From the argument in section 2, one finds that the near-horizon geometry of (6.4) does not contain $A d S$. However, (6.4) is obtained by dimensional reduction from an un-boosted nondilatonic black string, namely, from (5.6) with $\sigma=0$ and $r_{2}^{d}=Q$. (This is also a special

\footnotetext{
${ }^{9}$ This corrects typos in 17.
} 
case of (6.2) with $\xi=1 / 2, p=1$ ). Then as discussed above, the discussion on the greybody factors again reduces to that on the near-horizon BTZ geometry.

Although the argument in section 3 becomes singular when $\sigma=0$, it can be applied to this case with appropriate modification. First, it follows from (6.1) that the BekensteinHawking entropy of (6.4) is equal to that of the black string (5.6) with $\sigma=0$. Second, the entropy of (5.6) with $\sigma=0$ and the radius of $x$ equal to $R$ is the same as that of (5.6) with $\sigma \neq 0$ and the Lorentz contracted radius $R^{\prime}=R / \cosh \sigma$. This means that the entropy of the black string, which is supposed to express the total number of the relevant states, is invariant under boosts. For more detailed discussions on the entropy and boosts, see [36]. Since by dimensional reduction the geometry (5.6) with $\sigma \neq 0$ gives the dyonic dilaton black holes (5.5), the argument in the previous section is applied to the boosted black string. To discuss the original entropy of (6.4), we have only to use $R^{\prime}$ in the previous argument. We remark that one cannot freely boost the black strings in the examples in the previous section since $\sigma$ 's were fixed by the relation $r_{\sigma}=r_{1, g}$. The condition (3.6) is satisfied when $Q \gg 1$. The entropy counting for $d=1$ case has been discussed in a somewhat different way in 11 .

Together with the previous argument on the Reissner-Nordstrom black holes, we now understand the connection between the BTZ black holes and the geometries with $(\xi, p)=(0,0)$ and $(1 / 2,0)$. Furthermore, since the geometries with different $p$ are related by dimensional reduction, it is possible to similarly discuss the entropy and the greybody factors for all the cases with

$$
\xi=0 \text { or } \frac{1}{2}
$$

In [17], the scalar wave equation for (6.2) has been analyzed and some features closely related to $A d S_{3}$ have been found. The discussion here explains their geometrical reason for $\xi=0,1 / 2$.

Starting from (2.11) with (5.2) and $\sigma=0$, dimensional reduction yields the black holes in string theory in $D=5$ and $D=4$ with two and three charges respectively 229. One can discuss the entropy and the greybody factors similarly to the case of (6.4). In fact, if all these charges are the same, the geometries become (6.4). The corresponding greybody factors have been discussed in [17]. The geometry in the $D=5$ case is closely related to the fundamental strings and the entropy in the extremal case has been discussed using the stretched horizon [37.

\section{$7 \quad$ Discussion}

We argued that under mild conditions if a black hole geometry has a global structure of the type of the non-extremal Reissner-Nordstrom black holes, its near-horizon geometry 
becomes $A d S_{2}$ times a sphere. In addition, if such a black hole can be lifted to a boosted black string, the near-horizon geometry of the latter contained a BTZ geometry. Because of these facts, the calculation of the Bekenstein-Hawking entropy and the greybody factors was essentially reduced to the corresponding calculation in the near-horizon BTZ geometry under appropriate conditions. Furthermore, using dimensional reduction, we showed that in some cases similar arguments hold even if the geometry does not contain $A d S$ near the horizon.

The arguments in this paper extend the results in the literature on the black hole thermodynamics using the near-horizon BTZ geometry [3]- [6], [10]- [12, [30]. In contrast to the cases discussed in the literature, ours include the geometries whose extremal limit is not supersymmetric as the Reissner-Nordstrom black holes in $D \neq 4,5$.

We also showed that the near-horizon wave equations discussed in [17 are nothing but the $s$-wave equation in the BTZ background and gave a geometrical explanation for the $A d S_{3}$ structure found in [17] in some cases. By further investigations, the connection to the BTZ black holes might be found also in the other cases.

Our results indicate that there exists a kind of universality for the Reissner-Nordstrom type black holes (2.7) as pointed out in some cases by [3]; the thermodynamics is effectively reduced to that of the BTZ black holes and its characteristics do not depend on details of each black hole. This is analogous to the no-hair theorem and the universal behavior of the absorption cross-sections found in [38, 17]. It is suggestive that the conformal diagrams for the BTZ black holes are similar to those of the Reissner-Nordstrom black holes.

Moreover, the CFT structure of the greybody factors discussed in section 4 and [17] suggests existence of the underlying CFT describing the black holes and $p$-branes. The nearhorizon BTZ geometry may give important clues. In particular, it would be very interesting to further investigate the string theory and quantum gravity in $A d S_{3}$ and $A d S_{2}$ [28] and the connection to the AdS/CFT correspondence [16] discussed in [14, 15].

Finally, for the black holes in (5.1) in the toroidally compactified string theory, the correspondence between the black holes and CFT's (including the AdS(BTZ)/CFT correspondence) have been extensively studied. The argument in section 4 implies that such a correspondence might be extended to the $N=2$ black holes in the Calabi-Yau compactification. In this case, we have the exactly solvable CFT describing the string theory, i.e., Gepner models. It may be another interesting problem to explore the $N=2$ black holes in relation to the near-horizon BTZ black holes and the Gepner models. 


\section{Acknowledgements}

I would like to thank V. Balasubramanian, S.S. Gubser, K. Hori, A.W. Peet and D. Waldram for useful discussions and/or conversations. I would also like to thank the Aspen Center for Physics, where part of this work was done, for its hospitality. This work was supported in part by Japan Society for the Promotion of Science.

\section{Appendix}

\section{A.1 result of $K_{a b c d}$}

Using computers, it is easy to calculate the tensor defined in (2.2). The non-vanishing independent components for the geometry (2.1) are

$$
\begin{aligned}
K_{\text {trtr }}= & \frac{1}{4} r^{-(2+d)}(r / q)^{\alpha d}[-d(\alpha-1)\{d(\alpha-2)+2(\beta-1)\} \mu \\
& \left.+\alpha d\{\alpha d+2(\beta-1)\} r^{d}+4 k q^{2 \beta} r^{2(1-\beta)+d}\right], \\
K_{t y_{i} t y_{i}}= & \frac{1}{4} r^{-2(1+d)}(r / q)^{2(\alpha d+\beta)}\left[\alpha(\alpha-1) d^{2} \mu^{2}+\alpha(1-2 \alpha) d^{2} \mu r^{d}\right. \\
& \left.-4 k q^{2 \beta} \mu r^{2(1-\beta)+d}+\alpha^{2} d^{2} r^{2 d}+4 k q^{2 \beta} r^{2(1-\beta+d)}\right], \\
K_{y_{i} r y_{i} r}= & \frac{1}{4} r^{-2}\left(r^{d}-\mu\right)^{-1}(r / q)^{\alpha d}[\alpha d\{2(\beta-1)+d(\alpha-1)\} \mu \\
K_{y_{i} y_{j} y_{i} y_{j}}= & \frac{1}{4} r^{-(2+d)}(r / q)^{2(\alpha d+\beta)}\left[\alpha^{2} d^{2}\left(\mu-r^{d}\right)-4 k q^{2 \beta} r^{2(1-\beta)+d}\right] \quad(i \neq j),
\end{aligned}
$$

with $\mu=r_{0}^{d}$. We have only $K_{t r t r}$ for $p=0$ and $K_{t r t r}, K_{t y t y}$ and $K_{y r y r}\left(y_{1} \equiv y\right)$ for $p=1$.

\section{A.2 $\quad A d S_{2}, A d S_{3}$ and the BTZ geometry}

Here we briefly summarize the geometries of $A d S_{2}, A d S_{3}$ and the BTZ black holes and their relation.

$A d S_{2+p}$ is defined by

$$
\begin{aligned}
& -x_{0}^{2}-x_{1}^{2}+\sum_{i=2}^{p+1} x_{i}^{2}=-l^{2} \\
& d s^{2}=-d x_{0}^{2}-d x_{1}^{2}+\sum_{i=2}^{p+1} d x_{i}^{2} .
\end{aligned}
$$

A part of $A d S_{2}$ is parametrized by

$$
x_{0}=\sqrt{r^{2}-l^{2}} \sinh \theta, \quad x_{1}=r, \quad x_{2}=\sqrt{r^{2}-l^{2}} \cosh \theta .
$$


Then the metric takes the form

$$
\begin{aligned}
d s^{2} & =-\left(r^{2}-l^{2}\right) d \theta^{2}+\left[(r / l)^{2}-1\right]^{-1} d r^{2} \\
& =l^{2}\left[-(u-1) d \theta^{2}+\frac{1}{4} \frac{d u^{2}}{u(u-1)}\right] \\
& =l^{2}\left[-4 z(z-1) d \theta^{2}+\frac{d z^{2}}{z(z-1)}\right] .
\end{aligned}
$$

Here we have defined $u$ and $z$ by $u=r^{2} / l^{2}$ and $u-1=4 z(z-1)$ respectively.

To parametrize $\operatorname{Ad}_{3}(S L(2, R))$, the following forms of the metric are often used,

$$
\begin{aligned}
d s^{2} & =-\cosh ^{2} \mu d \theta_{+}^{2}+d \mu^{2}+\sinh ^{2} \mu d \theta_{-}^{2} \\
& =\frac{l^{2}}{\omega^{2}}\left(d \omega^{2}-d x^{+} d x^{-}\right) \\
& =-\left[(r / l)^{2}-1\right] d \theta_{+}^{2}+\left[(r / l)^{2}-1\right]^{-1} d r^{2}+(r / l)^{2} d \theta_{-}^{2} .
\end{aligned}
$$

In terms of $S L(2, R)$, these correspond respectively to the parametrization of $g \in S L(2, R)$,

$$
g=g_{0}\left(\theta_{L}\right) g_{1}(2 \mu / l) g_{0}\left(\theta_{R}\right), \quad g_{3}\left(x^{-} / l\right) g_{2}(\tau) s g_{3}\left(x^{+} / l\right), \quad g_{2}\left(\theta_{L}\right) g_{1}(\rho) g_{2}\left(-\theta_{R}\right),
$$

and $d s^{2}=\left(l^{2} / 2\right) \operatorname{Tr}\left(g d g^{-1} g d g^{-1}\right)$, where

$$
\begin{aligned}
& g_{0}(t)=\left(\begin{array}{cc}
\cos t / 2 & \sin t / 2 \\
-\sin t / 2 & \cos t / 2
\end{array}\right), \quad g_{1}(t)=\left(\begin{array}{cc}
\cosh t / 2 & \sinh t / 2 \\
\sinh t / 2 & \cosh t / 2
\end{array}\right), \\
& g_{2}(t)=\left(\begin{array}{cc}
e^{t / 2} & 0 \\
0 & e^{-t / 2}
\end{array}\right), \quad g_{3}(t)=\left(\begin{array}{ll}
1 & 0 \\
t & 1
\end{array}\right), \quad s=\left(\begin{array}{cc}
0 & 1 \\
-1 & 0
\end{array}\right),
\end{aligned}
$$

$\theta_{ \pm}=(l / 2)\left(\theta_{L} \pm \theta_{R}\right), \omega=l e^{-\tau / 2}$ and $r^{2}=l^{2} \cosh ^{2}(\rho / 2)$.

These parametrizations are closely related to the $S L(2, R)$ representations in the elliptic, parabolic and hyperbolic basis, respectively [23]. The scalar wave functions in each coordinate system are nothing but the matrix elements of the representations in the corresponding basis. They are well-classified in terms of the representation theory and typically expressed by the Jacobi polynomials, the Bessel functions and the hypergeometric functions, respectively.

To get the BTZ black hole geometry, the parametrization (A.9) is used. By making the changes of variables

$$
\frac{r^{2}}{l^{2}}=\frac{\rho^{2}-\rho_{-}^{2}}{\rho_{+}^{2}-\rho_{-}^{2}}, \quad\left(\begin{array}{c}
\theta_{+} \\
\theta_{-}
\end{array}\right)=\frac{1}{l}\left(\begin{array}{cc}
\rho_{+} & -\rho_{-} \\
-\rho_{-} & \rho_{+}
\end{array}\right)\left(\begin{array}{c}
\tau / l \\
\varphi
\end{array}\right),
$$

and identifying $\varphi$ to $\varphi+2 \pi$, one obtains the BTZ metric (2.16). 
$A d S_{2}$ is also related to $A d S_{3}$. By dimensionally reducing (A.9) with respect to $\theta_{L}$ and making a change of variables $(r / l)^{2}=z$, one obtains the $A d S_{2}$ metric (A.6) up to a factor [27, 28.

\section{A.3 dimensional reduction and scalar wave equations}

We consider a $(D+1)$-dimensional geometry of the form

$$
d s_{D+1}^{2}=F(r)\left[-d t^{2}+d x^{2}+u(r)(\cosh \sigma d t-\sinh \sigma d x)^{2}\right]+g_{r r}(r) d r^{2}+g_{\Omega}(r) d \Omega_{d+1}^{2},
$$

where $D=d+3$ and $\sigma$ corresponds to the boost parameter. By dimensional reduction (in Einstein frame) with respect to $x$, one obtains the $D$-dimensional geometry

$$
d s_{D}^{2}=\left(F h_{\sigma}\right)^{1 /(d+1)}\left[-F h_{\sigma}^{-1} f d t^{2}+g_{r r} d r^{2}+g_{\Omega} d \Omega_{d+1}^{2}\right]
$$

where $h_{\sigma}(r)=1+u(r) \sinh ^{2} \sigma$ and $f(r)=1-u(r)$.

A scalar field in $D+1$ dimensions is expanded as $\Phi_{D+1}=\sum e^{-i \omega t+i k x} \phi_{m}(r) \chi_{m}$ where $\chi_{m}$ is the eigenfunction of the Laplacian on $S^{d+1}$ with the eigenvalue $-\Lambda=-m(m+d)$. The momentum $k$ becomes the Kaluza-Klein charge in $D$ dimensions. The radial equation is

$$
\left[\left\{\left(\omega^{2}-k^{2}\right)+u(\omega \sinh \sigma-k \cosh \sigma)^{2}\right\}+\sqrt{f / \bar{g}} \partial_{r}\left(F \sqrt{f \bar{g}} g^{r r}\right) \partial_{r}-F f g_{\Omega}^{-1} \Lambda\right] \phi_{m}(r)=0
$$

where $\bar{g}=g_{r r} g_{\Omega}$. On the other hand, an uncharged scalar field in $D$ dimensions is expanded as $\Phi_{D}=\sum e^{-i \omega t} \phi_{m}(r) \chi_{m}$ and the radial wave equation is

$$
\left[\omega^{2} h_{\sigma}+\sqrt{f / \bar{g}} \partial_{r}\left(F \sqrt{f \bar{g}} g^{r r}\right) \partial_{r}-F f g_{\Omega}^{-1} \Lambda\right] \phi_{m}(r)=0
$$

As shown for the $D=5$ and 4 charged black holes [13], (A.15) takes the same form as (A.16) in terms of the new variables

$$
\omega^{\prime 2}=\omega^{2}-k^{2}, \quad e^{ \pm \sigma^{\prime}}=e^{ \pm \sigma} \frac{\omega \mp k}{\omega^{\prime}} .
$$

Therefore, if $\Phi_{D+1}$ is independent of $x(k=0)$, the primed variables are the same as unprimed ones and hence the radial equation is invariant under the dimensional reduction (as it should be). Moreover, since (A.15) is regarded as the equation for the charged scalars in $D$ dimensions, the discussion on the uncharged scalars in section 4 can be extended to the charged case by appropriate changes of parameters. In this case, the parameters of the corresponding BTZ black holes effectively change according to (A.17). 


\section{References}

[1] M. Bañados, C. Teitelboim and J. Zanelli, Phys. Rev. Lett. 69 (1992) 1849, hep-th/9204099; M. Bañados, M. Henneaux, C. Teitelboim and J. Zanelli, Phys. Rev. D48 (1993) 1506, gr-qc/9302012.

[2] S. Carlip, Class. Quant. Grav 12 (1995) 2853, gr-qc/9506079.

[3] S. Hyun, hep-th/9704005.

[4] K. Sfetsos and K. Skenderis, Nucl. Phys. B517 (1998) 179, hep-th/9711138.

[5] A. Strominger, J. High Energy Phys. 02 (1998) 009, hep-th/9712251.

[6] V. Balasubramanian and F. Larsen, Nucl. Phys. B528 (1998) 229, hep-th/9802198.

[7] S. Carlip, Phys. Rev. D51 (1995) 632.

[8] D. Birmingham, I. Sachs and S. Sen, Phys. Lett. 424B (1998) 275, hep-th/9801019.

[9] S. Carlip, hep-th/9806026.

[10] E. Teo, Phys. Lett. 430B (1998) 57, hep-th/9803064;

N. Kaloper, Phys. Lett. 434B (1998) 285, hep-th/9804062;

M. Cvetič and F. Larsen, hep-th/9805097; hep-th/9805146.

[11] M.Z. Iofa and L.P. Zayas, Phys. Lett. 434B (1998) 264, hep-th/9803083.

[12] M.Z. Iofa and L.P. Zayas, hep-th/9804129.

[13] J. Maldacena and A. Strominger, Phys. Rev. D55 (1996) 861, hep-th/9609026;

S.S. Gubser and I.R. Klebanov, Phys. Rev. Lett. 77 (1996) 4491, hep-th/9609076.

[14] E. Teo, hep-th/9805014.

[15] H.J.W. Müller-Kristen, N. Ohta and J.-G. Zhou, hep-th/9809193.

[16] J. Maldacena, hep-th/9711200;S.S. Gubser, I.R. Klebanov and A.M. Polyakov, Phys. Lett. 428B (1998) 105, hep-th/9802109; E. Witten, hep-th/9802150.

[17] Y. Satoh, Phys. Rev. D58 (1998) 044004, hep-th/9801125.

[18] G.W. Gibbons and P.K. Townsend, Phys. Rev. Lett. 71 (1993) 3754, hep-th/9307049;M.J. Duff, G.W. Gibbons and P.K. Townsend, Phys. Lett. 332B (1994) 321, hep-th/9405124.

[19] G.W. Gibbons, G. Horowitz and P.K. Townsend, Class. Quant. Grav 12 (1995) 297, hep-th/9410073.

[20] V. Balasubramanian, P. Kraus and A. Lawrence, hep-th/9805171.

[21] E. Keski-Vakkuri, hep-th/9808037.

[22] J. Maldacena and A. Strominger, hep-th/9804085. 
[23] N.Ja. Vilenkin and A.U. Klimyk, Representation of Lie Groups and Special Functions, (Kluwer Academic Publishers, Dordrecht, 1991).

[24] M. Natsuume and Y. Satoh, Int. J. Mod. Phys. A13 (1998) 1229, hep-th/9611041.

[25] J. Maldacena and A. Strominger, Phys. Rev. D56 (1997) 4975, hep-th/9702015.

[26] D. Birmingham, I. Sachs and S. Sen, Phys. Lett. 413B (1997) 281, hep-th/9707188.

[27] D.A. Lowe and A. Strominger, Phys. Rev. Lett. 73 (1994) 1468, hep-th/9403186.

[28] A. Strominger, hep-th/9809027.

[29] D. Youm, Black holes and solitons in string theory, hep-th/9710046.

[30] H.W. Lee and Y.S. Myung, Phys. Rev. D58 (1998) 104013, hep-th/9804095.

[31] M. Cvetič and F. Larsen, Phys. Rev. D56 (1997) 4994, hep-th/9705192; Nucl. Phys. B506 (1997) 107, hep-th/9706071.

[32] D. Kastor and K.Z. Win, Phys. Lett. 411B (1997) 33, hep-th/9705090;

K. Behrndt, M. Cvetič and W. Sabra, Phys. Rev. D58 (1998) 084018, hep-th/9712221.

[33] G.W. Gibbons and K. Maeda, Nucl. Phys. B298 (1998) 741.

[34] M.J. Duff, H. Lü and C.N. Pope, Phys. Lett. 382B (1996) 73, hep-th/9604052.

[35] G.T. Horowitz and A. Strominger, Nucl. Phys. B360 (1991) 197.

[36] S.R. Das, S.D. Mathur, S.K. Rama and P. Ramadevi, Nucl. Phys. B527 (1998) 187, hep-th/9711003;G.T. Horowitz and E.J. Martinec, Phys. Rev. D57 (1998) 4935, hep-th/9710217.

[37] A.W. Peet, Nucl. Phys. B456 (1995) 732, hep-th/9506200; A. Sen, Mod. Phys. Lett. A10 (1995) 2081,hep-th/9504147.

[38] S. Das, G. Gibbons and S. Mathur, Phys. Rev. Lett. 78 (1997) 417, hep-th/9609052. 